\title{
Neurogenic Pulmonary Edema Following Status Epilepticus: An Unusual Case
}

\author{
Aradhana Aneja $^{\mathrm{a}, \mathrm{d}}$, Niraj Arora ${ }^{\mathrm{b}}$, Ramkrishna Sanjeeva ${ }^{\mathrm{a}}$, Kapil Semalti ${ }^{\mathrm{c}}$
}

\begin{abstract}
Neurogenic pulmonary edema (NPE) is rare in pediatric patients. It occurs most commonly following brain malignancies, traumatic brain injuries, infections and seizures. It has rarely been reported in pediatric patients following status epilepticus. The pathophysiology is not completely understood and is proposed to be due to overactivity of sympathetic nervous system leading to pulmonary interstitial and alveolar edema. Here we present a case of a 4-year-old healthy boy with status epilepticus secondary to febrile seizures who developed NPE. Treatment is supportive ventilator management with low tidal volume and high positive end-expiratory pressure. Timely diagnosis and management is the key for good recovery. It should be considered in the differential diagnosis of patients with rapidly developing pulmonary edema in the setting of normal cardiac function.
\end{abstract}

Keywords: Neurogenic pulmonary edema; Status epilepticus; Respiratory distress

\section{Introduction}

Neurogenic pulmonary edema (NPE) could be a contributing factor to morbidity after status epilepticus. It was first described by Shanahan et al in 1908 following epileptic seizures but still after hundred years, it remains an under-appreciated entity in the clinical world [1]. It manifests as an acute onset of pulmonary edema in the setting of central nervous system injury without previous cardiac pathology [2]. In adults, NPE

Manuscript accepted for publication November 20, 2015

aDepartment of Paediatrics, Base Hospital, Delhi Cantonment, New Delhi 110010, India

bDepartment of Neurology, Drexel University College of Medicine, $245 \mathrm{~N}$ 15th Street, Philadelphia, PA 19102, USA

'Department of Radiology, Army hospital-Research and Referral, Delhi Cantonment, New Delhi, 110010, India

${ }^{\mathrm{d} C}$ Corresponding Author: Aradhana Aneja, Department of Paediatrics, Base Hospital, Delhi Cantonment, New Delhi 110010, India.

Email: aradhanaaneja@gmail.com

doi: http://dx.doi.org/10.14740/ijcp230w could result from various central nervous system disorders such as subarachnoid hemorrhage following aneurysm rupture, brain malignancies, traumatic brain injuries, infections and prolonged seizure activity [3]. It develops within minutes, hours or days following injury and significantly complicates the overall clinical status of the patient [4]. In pediatric patients, respiratory compromise has been attributed to anticonvulsant drug use and only isolated case reports exist for its association with recurrent seizures $[3,5,6]$. This is likely due to lack of awareness about the diagnosis and unpredictable nature of its etiology $[2,3]$. The diagnosis of NPE requires high index of clinical suspicion. We report a case of a 4-yearold healthy boy, known case of simple febrile seizures, who presented with febrile status epilepticus and developed NPE. Strong clinical suspicion and prompt management resulted in complete recovery with no morbidity. The case highlights the importance of considering NPE in the differential diagnosis of respiratory compromise after pediatric status epilepticus.

\section{Case Report}

A 4-year-old developmentally normal boy was a known case of simple febrile seizure, with onset at age of 1.5 years. He presented with history of fever of 1 day duration followed by history of generalized tonic-clonic seizures for $2 \mathrm{~h}$. Detailed clinical examination revealed temperature of $101^{\circ} \mathrm{F}$, tachycardia with heart rates in range of $140-160 / \mathrm{min}$, and tachypnea with subcostal retractions. However, rest of the systemic examination was within normal limits. Initially, child was managed as per protocol for status epilepticus, i.e. supportive management for airway, breathing and circulation and given intravenous lorazepam as antiepileptic agent. Seizure was not controlled with two doses of lorazepam, so child was loaded with intravenous phenytoin. As the seizure activity continued, intravenous valproic acid was given and the child was intubated for airway protection. Finally seizure responded to IV midazolam infusion.

After seizure control, child developed features of respiratory distress in form of tachypnea, tachycardia and low $\mathrm{SpO}_{2}$ (86-88\%). Child was immediately put on mechanical ventilation. The lung compliance was poor and child required high pressures and high positive end-expiratory pressure to expand the lungs. There was clinical suspicion of acute respiratory distress syndrome. Routine chest X-ray revealed diffuse bilateral 


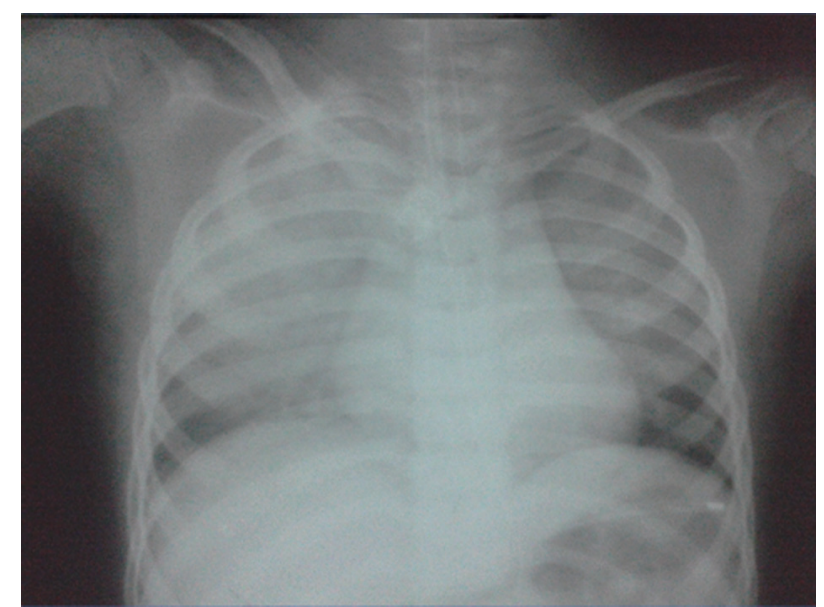

Figure 1. Chest $\mathrm{X}$-ray revealing bilateral diffuse pulmonary infiltrates with normal sized heart.

infiltrates on both lung fields (right $>$ left) with normal sized heart (Fig. 1). Arterial blood gas (ABG) analysis revealed severe respiratory acidosis with partial compensation $(\mathrm{pH} 6.9$, $\mathrm{pCO}_{2}: 147, \mathrm{pO}_{2}: 90.3$ on $\mathrm{FiO}_{2}$ of 0.5 ). On the basis of above $\mathrm{ABG}$ revealing $\mathrm{paO}_{2} / \mathrm{FiO}_{2}$ of $180 \mathrm{~mm} \mathrm{Hg}$ (normal $>500 \mathrm{~mm}$ $\mathrm{Hg}$ ) and characteristic chest X-ray findings, child was diagnosed as a case of acute respiratory distress syndrome, likely etiology being aspiration. Other laboratory investigations revealed hemoglobin of $12.0 \mathrm{~g} / \mathrm{dL}$, leucocytosis with neutro-

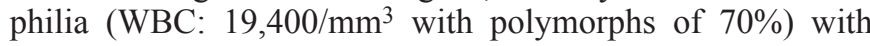
peripheral blood smear showing no features of sepsis, and the biochemical parameters including serum albumin and serum transaminases were within normal limits.

Child was managed with restricted IV fluids, mechanical ventilation and empirically started on broad spectrum antibiotics (ceftriaxone and amikacin) and antimalarial (artesunate). After $6 \mathrm{~h}$ of admission, child had episodes of sudden desaturation on mechanical ventilation and pink frothy secretions were seen in the endotracheal tube. Endotracheal suctioning revealed frank blood stained secretions. Detailed clinical examination revealed raised jugular venous pressure, serial recordings of high blood pressure (118 - 140/78 - $90 \mathrm{~mm} \mathrm{Hg}$ ) and bilateral crepitations in both lung fields. Child developed edema and hepatomegaly. The ventilator settings were further changed in view of acute respiratory distress syndrome with likely NPE. The child was managed with high positive endexpiratory pressures up to 15 , low tidal volume $(5 \mathrm{~mL} / \mathrm{kg})$, optimal $\mathrm{FiO}_{2}$ and restricted maintenance fluids. The urine output improved $(2-3 \mathrm{~mL} / \mathrm{kg} / \mathrm{h})$ and child started improving after 12 $\mathrm{h}$ and the child was weaned off the ventilator. The child was extubated after $36 \mathrm{~h}$ of admission and completely recovered within in $72 \mathrm{~h}$. The child is on follow-up and with no cardiovascular/neurogenic sequelae.

\section{Discussion}

NPE is an increase in pulmonary interstitial and alveolar fluid that is due to an acute central nervous system injury and usually develops rapidly after the injury [2]. Sympathetic overactivity in hypothalamus, medulla, nuclei of solitary tract and area postrema after central nervous system injury is a proposed mechanism [7]. The downstream effects of sympathetic overactivity vary depending on the cardiopulmonary effects [2]. Pathologically, there is marked pulmonary vascular congestion with perivascular edema, extravasation and intra-alveolar accumulation of protein rich edema fluid and intra-alveolar hemorrhage [8]. The clinical pathology overlaps with acute lung injury and acute respiratory distress syndrome [6]. It is largely underdiagnosed and poorly reported because of the poor clinical status of the patient caused by the primary central nervous system injury and it necessitates rapid and often complex treatment [9].

Clinically, NPE presents in minutes to hours (immediate onset) of a severe central nervous system insult such as subarachnoid hemorrhage, traumatic brain injury or status epilepticus. However, presentation in hours to days (delayed onset) has been described [2]. The signs of NPE are quite non-specific [3, 9]. It presents subjectively with a sudden onset of dyspnea, chest pain, worsening of expectoration, vomiting and weakness. Clinical examination reveals tachypnea, tachycardia, basal bilateral pulmonary crackles, respiratory distress or failure, expectoration of sanguinolent sputum or even hemoptysis, hypoxemia and increased systemic blood pressure [10]. The most relevant imaging method is the chest X-ray examination that reveals a normal size heart with diffuse bilateral alveolar opacities [9]. Although the levels of some substances such as brain natriuretic peptide, blood C-reactive protein and IL-6 are increased, unfortunately none of these can be used as a marker specific for NPE [10]. It is proposed that measurement of serum catecholamines might be helpful and treatment with $\alpha$-adrenergic blocking agent, such as phentolamine, may be considered [2].

In this case, a 4-year-old child developed respiratory distress following status epilepticus and clinical evaluation revealed features of pulmonary edema. The differential diagnosis included acute respiratory distress syndrome due to aspiration and cardiogenic pulmonary edema. The cardiac examination and workup was within normal limits. There was no evidence of acute infection and the blood cultures for bacteria, fungus and viruses were also negative. Cardiac functions were also within normal range. In pulmonary edema of unknown origin, associated with neurological condition, the possibility of NPE should be considered.

The case was reported to highlight the importance of strong clinical suspicion and prompt management of the condition. So far, there is only one reported case of NPE following febrile status epilepticus in this age group [3]. Such cases are rare and need constant vigilant management for speedy recovery. Our patient received timely supportive treatment and recovered completely from the event. Early recognition and appropriate use of positive end-expiratory pressure and judicious fluid management are important in the management of such patients.

\section{Conclusion}

NPE should be considered in the differential diagnosis for the rapid progression of respiratory failure following an acute neu- 
rological injury such as status epilepticus in a child. The associated mortality rate is high, but recovery is usually rapid with early and appropriate management. Prompt respiratory support and treatment of acute neurological insult can prevent further cerebral hypoxemic injury. The treatment of NPE should aim to meet the oxygenation needs without impairing cerebral hemodynamic, to avoid pulmonary worsening and to treat possible associated myocardial dysfunction. Treatment is based primarily on ventilation with positive end-expiratory pressure and support of the patient's general health status. Recovery is usually very rapid, and $52.4 \%$ of patients recovered within 72 $\mathrm{h}$ [11], if managed promptly and appropriately.

\section{Conflict of Interest}

None.

\section{References}

1. Shanahan W. Acute pulmonary edema as a complication of epileptic seizures. NY Med J. 1908;37:54-56.

2. Davison DL, Terek M, Chawla LS. Neurogenic pulmonary edema. Crit Care. 2012;16(2):212.

3. Reuter-Rice K, Duthie S, Hamrick J. Neurogenic pulmonary edema associated with pediatric status epilepticus. Pediatr Emerg Care. 2011;27(10):957-958.

4. Friedman JA, Pichelmann MA, Piepgras DG, McIver JI,
Toussaint LG, 3rd, McClelland RL, Nichols DA, et al. Pulmonary complications of aneurysmal subarachnoid hemorrhage. Neurosurgery. 2003;52(5):1025-1031; discussion 1031-1022.

5. Mulroy JJ, Mickell JJ, Tong TK, Pellock JM. Postictal pulmonary edema in children. Neurology. 1985;35(3):403405.

6. Nguyen TT, Hussain E, Grimason M, Goldstein J, Wainwright MS. Neurogenic pulmonary edema and acute respiratory distress syndrome in a healthy child with febrile status epilepticus. J Child Neurol. 2013;28(10):12871291.

7. Colice GL. Neurogenic pulmonary edema. Clin Chest Med. 1985;6(3):473-489.

8. Kandatsu N, Nan YS, Feng GG, Nishiwaki K, Hirokawa M, Ishikawa K, Komatsu T, et al. Opposing effects of isoflurane and sevoflurane on neurogenic pulmonary edema development in an animal model. Anesthesiology. 2005;102(6):1182-1189.

9. Sedy J, Zicha J, Kunes J, Jendelova P, Sykova E. Mechanisms of neurogenic pulmonary edema development. Physiol Res. 2008;57(4):499-506.

10. Baumann A, Audibert G, McDonnell J, Mertes PM. Neurogenic pulmonary edema. Acta Anaesthesiol Scand. 2007;51(4):447-455.

11. Fontes RB, Aguiar PH, Zanetti MV, Andrade F, Mandel M, Teixeira MJ. Acute neurogenic pulmonary edema: case reports and literature review. J Neurosurg Anesthesiol. 2003;15(2):144-150. 